\title{
Two Theorem Tables of Matrix Algebra
}

\author{
By Gilbert C. Best
}

Abstract. It is shown in this paper how it is possible, by a slight modification of the mode of presentation, to state many of the theorems of matrix algebra in table form. Due to the large number of theorems presented the tables give only the statement of each theorem. It is not considered feasible to also attempt to indicate sources and proofs.

1. Introduction. Many theorems are of such a nature that they can be stated in tạble form. Take, for example, the theorem which reads, "If $A$ is a non-singular symmetric matrix then so is $A^{-1}$." As a first step we restate this in "operator" form. We define the "inverse-forming" operator $O^{-1}$ to be such that $O^{-1} A=A^{-1}$, where $A$ is assumed non-singular. Then the theorem reads, "The property of symmetry is invariant for the operator $O^{-1}$." Finally, defining " $v$ " to indicate invariance, the theorem may be presented in an "operator vs. property" table by a $v$ in row $O^{-1}$ and column "symmetry." This theorem appears in Table I in row 3 column 6 . This method of presentation has the obvious advantage of considerable compactness.

Again, consider the theorem

$$
\left(A^{T}\right)^{-1}=\left(A^{-1}\right)^{T} .
$$

Defining a "transposing" operator $O^{T}$ such that $O^{T} A=A^{T}$ this theorem can be rewritten in "operator" form as :

$$
O^{r} O^{-1} A=O^{-1} O^{r} A
$$

The theorem can then be stated as: "The operators $O^{T}$ and $O^{-1}$ are commutative." In this last form the theorem in question is readily presented in an "operator vs. operator" table by defining $k$ to indicate commutativeness. Then ( 1 ) is given as a $k$ in row $O^{T}$ and column $O^{-1}$, or, equally well, by a $k$ in row $O^{-1}$ and column $O^{T}$.

2. Operators. In general, any transformation of a matrix may be considered to define an operator. Also, for implied given fixed coefficients $\alpha_{1}, \cdots \alpha_{m}$ and an ordered set $\left(A_{1}, A_{2}, \cdots, A_{m}\right)$ of matrices, the linear combination $\sum_{i=1}^{m} \alpha_{i} A_{i}$ may be considered an operator. Similarly, any function of a matrix or matrices may be considered an operator. For example, the forming of the determinant, the extraction of the characteristic roots or characteristic vectors, or the product of two matrices, may all be considered operators. A numbered list of the operators used in this paper is shown below. The operators in the rows of Table 1 and the rows and columns of Table 2 preserve the same numbering.

3. Properties. The idea of a property also may be generalized beyond the wellknown and clearly defined properties such as symmetry, skew-symmetry, singularity, positive definiteness, etc. For example, the fact that a given matrix $A$ has specific roots, e.g., $2,-5,7$ say, is a property, and this property is unaffected by a

Received April 22, 1960. 
List OF OPERATORS

\begin{tabular}{|c|c|c|}
\hline * & Symbol & Explanation \\
\hline $\boldsymbol{0}$ & $I$ & Identity Operator \\
\hline 1 & Or & Transposer \\
\hline 2 & $\delta$ & Conjugator, i.e., sends $A=\left(a_{i j}\right)$ into $\bar{A}=\left(a_{i j}\right)$ \\
\hline$\overline{3}$ & $0^{-1}$ & Inverse Former (if inverse exists) \\
\hline 4 & $O^{4}$ & Adjoint Former (or adjugate, see [1]) \\
\hline 5 & $O^{(k)}$ & $k$ th Compound Former, see [1] \\
\hline 6 & $P Q$ & Equivalent Matrix Former* \\
\hline 7 & $P P_{T}^{T}$ & Congruent Matrix Former* \\
\hline 8 & $P \bar{P}^{T}$ & Hermitely Congruent Matrix Former* \\
\hline 9 & $P P^{-1}$ & Similar Matrix Former* \\
\hline 10 & $P P \perp$ & Orthogonally Similar Matrix Former* \\
\hline 11 & $U U^{\perp}$ & Unitarily Similar Matrix Former* \\
\hline 12 & $\sum$ & Sum Former \\
\hline 13 & $O^{\alpha}$ & Former of Product with Scalar $\alpha \neq 0$ \\
\hline 14 & LNCO & Former of Linear Combination (coefficients assumed fixed) \\
\hline 15 & $\pi$ & Former of Product of Matrices \\
\hline 16 & $\mathrm{O}^{\mathbf{P}}$ & Former of Matrix Raised to Power $P$ \\
\hline 17 & POL & $\begin{array}{l}\text { Former of a Polynomial from a Matrix (coefficients as- } \\
\text { sumed fixed) }\end{array}$ \\
\hline 18 & FUNC & Function Taker* \\
\hline 19 & $D E T$ & Determinant Taker \\
\hline 20 & $\operatorname{tr}$ & Trace Taker \\
\hline 21 & $\boldsymbol{R}$ & Characteristic Root Taker \\
\hline 22 & $\boldsymbol{V}$ & Characteristic Vector Taker \\
\hline
\end{tabular}

* Operator matrices or coefficients are over the same field as the matrix $A$ to which they are applied unless otherwise indicated.

\section{List of Properties}

\begin{aligned} & \\ & \hline 1 Piagonal \\ & 2 Block Diagonal (also called "direct sum") \\ & 3 Triangular. 2 types* : upper and lower \\ & 4 Block Triangular. 2 types* : upper and lower \\ & 5 Non-Singular \\ & 6 Symmetric \\ & 7 Hermitian \\ & 8 Skew Symmetric \\ & 9 Skew Hermitian \\ & 10 Orthogonal \\ & 11 Unitary \\ & 12 Normal \\ & 13 Diagonable, i.e., similar to a diagonal matrix \\ & 14 Symmetric Positive Definite (real) \\ & 15 Hermitian Positive Definite \\ & 16 Definite. 4 types* positive definite, positive semi-definite, negative \\ & semi-definite, negative definite \\ & 17 Specific Rank \\ & 18 Specific Index \\ & 19 Specific Invariant Factors \\ & 20 Specific Characteristic Equation or Characteristic Roots \\ & 21 Specific Characteristic Vectors \\ & 22 Specific Determinant \\ & * in Table 1 implies that the matrix remains the same type. \end{aligned}


similarity transformation. If a matrix has a specific determinant, rank, or index, that is also a property. A list of properties used is shown below, and the columns of Table 1 preserve the same numbering.

4. Rules of Application. If an operator is applied to more than one matrix at a time the following rules hold:

(1) If the operator $O$ is applicable to a single matrix, i.e., is a matrix-to-matrix operator, and $O$ is applied to the ordered set $\left(A_{1}, A_{2}, \cdots, A_{m}\right)$, then one obtains $\left(O A_{1}, O A_{2}, \cdots, O A_{m}\right)$.

(2) If the operator $O$ is applicable to two matrices and yields one matrix and $O$ is applied to the ordered set $\left(A_{1}, A_{2}, \cdots, A_{m}\right)$, then $O$ is first applied to $A_{1}, A_{2}$ yielding $A_{2}{ }^{\prime}$, then $O$ is applied to $A_{2}{ }^{\prime} A_{3}$ yielding $A_{3}{ }^{\prime}$, etc.

If a set of matrices $\left(A_{1}, A_{2}, \cdots, A_{m}\right)$ is said to have a property $P$, then each matrix of the set has property $P$.

TABLE 1

Operators vs. Properties

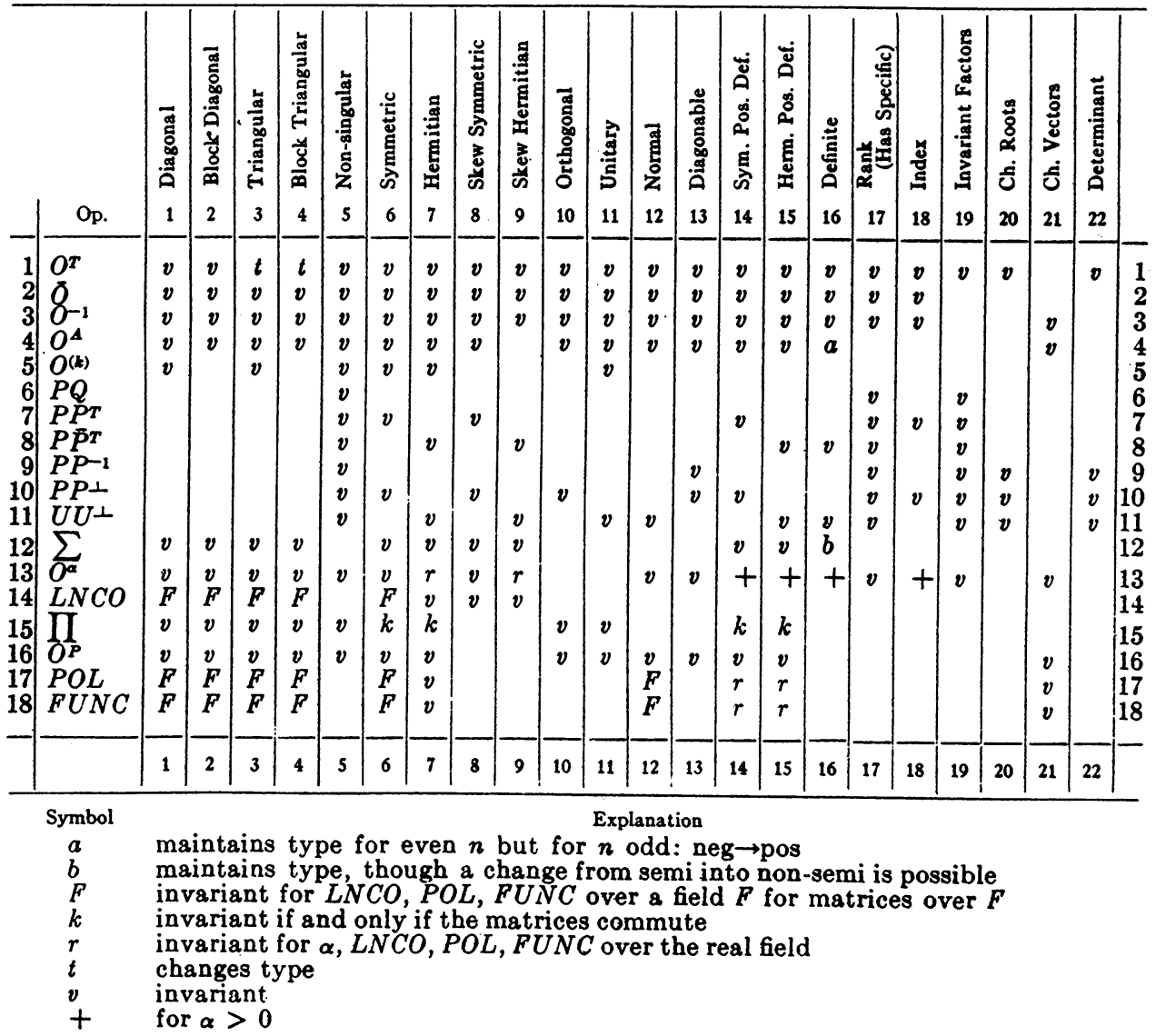


TABLE 2

Operators v8. Operators

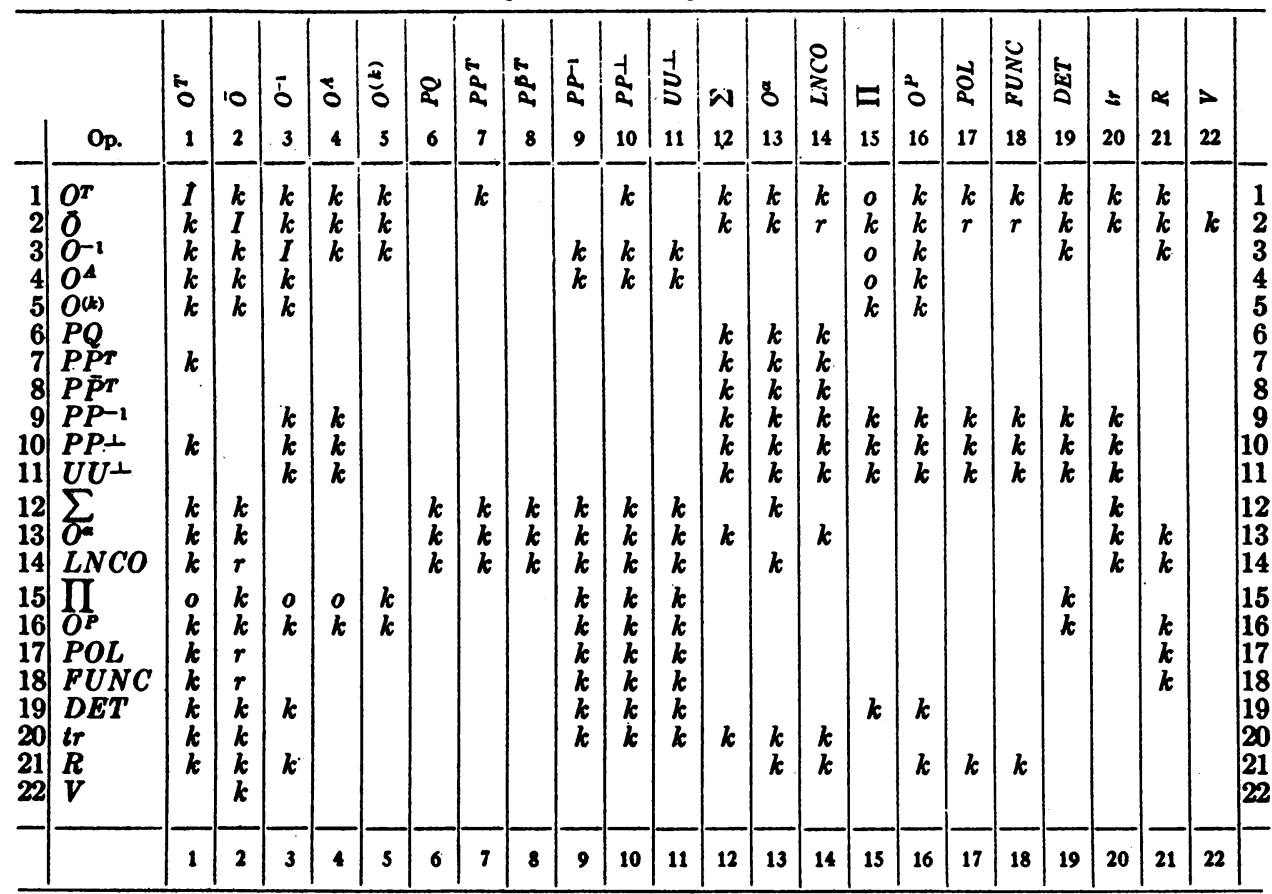

Symbol

Explanation

I two applications of operator equivalent to the identity operator

o order of product reverses, e.g. if $P=$ operator for row in table $P \Pi(A B)=$ $\prod P(B A)=P(B) * P(A)$

$k$ operators are commutative

$r$ operators are commutative for $L N C O, P O L, F U N C$ over the real field

3703 Tulsa Way

Fort Worth.7, Texas

1. A. C. AITren, Determinants and Matrices, Oliver \& Boyd, London, 1951, p. 51, p. 90. 